\title{
Feedback Alarm and Its Troubleshooting For Improvement of Microwave Link
}

\author{
Mohd Aamirullah Inamdar and Dr.Sayyad Ajij D \\ Department of Electronics \& Communication Engineering, MIT Aurangabad-431028.
}

\begin{abstract}
Initially NEC (Name of company) Microwave manufacturing, Japan is introduced briefly and then various parameters to establish microwave link is discussed then proposed system is explained. The flow chart to reduce the time of doing troubleshooting is explain. The main objective is to reduce the call drop of TATA DOCOMO and to overcome the drop if occur within short duration of time. There exists limited literature on NEC microwave, especially with regard to acknowledgments and troubleshooting. The working of NEC microwave, classification \& tools on the basis of the NEC equipment used in TATA DOCOMO \& flow chart to reduced the time is described. According to different types they produce different acknowledgment and depending upon the acknowledgment the troubleshooting strategy changes.

Index Terms: Troubleshooting, O\&M report, Acknowledgment, Proposed system
\end{abstract}

\section{Introduction}

\section{A. Introduction}

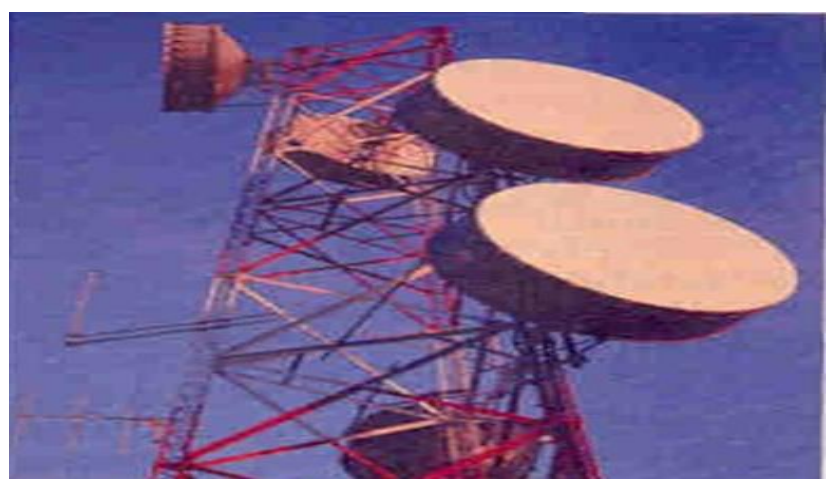

Today wireless technology is used in many applications well integrated into our everyday life. Planning a good, stable and reliable microwave network can be quite challenging. Careful planning and detailed analysis is required for a microwave radio system before the equipment can be installed. A poorly designed path can result in periodic system outages, resulting in increased system latency, decreased throughput, or worst case, a complete failure of the system. It is generally agreed that a microwave signal is a signal whose fundamental frequency is between $300 \mathrm{MHz}$ and $300 \mathrm{GHz}\left(1 \mathrm{GHz}=10^{9} \mathrm{~Hz}\right)[1,7]$. In terms of wavelength, a microwave signal has a wavelength between $0.1 \mathrm{~cm}$ and $100 \mathrm{~cm} A$ The waveguide is a hollow mechanical structure that permits propagation of microwave signals from one point to another with the least possible loss. most commonly used waveguides are those having a rectangular form. There are, however, a variety of rectangular waveguides, each being identified according to its internal dimensions. Each type of waveguide allows microwave propagation within a particular frequency band [2]. Discussing all the acknowledgment present in working link of NEC microwave and there trouble shooting methods.

\section{B. Classification Of NEC Microwave:}

1. $\mathrm{SDH}$ (Synchronous Digital Hierarchy)

2. PDH (Plesuchronous Digital Hierarchy)

SDH: Pasolink+ STM1, Pasolink Neoi

PDH: Pasolink CPV, Pasolink V4.

\section{Tools:}

The following tools are used in NEC Microwave

1) Software Tools

2) Hardware Tools 
1)Software Tools:- Four software's are used for commissioning of different types of NEC microwave and one software is used for observing all the sites from the server. The details are as follows.

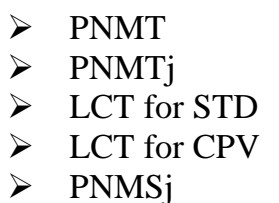

The PNMT is used for the commissioning of Passo+/Passo V4 IDU, PNMTj is used for observing the alarms of NEOi/Neo/ic in working link, LCT for STD is used for commissioning of NEOi IDU and LCT for CPV is used for commissioning of NEO/ic IDU. The PNMSj is used for the observation of all the types of NEC microwave from the server.

2)Hardware Tools:-Three hardware are used in this, the details are as follows

$>$ IDU

$>$ ODU

$>$ Antenna

IDU (In door Unit):-It is used for the assigning the frequency, power to ODU for microwave link. It is also used for observing the alarms in working Link.

ODU (Out Door Unit):-It is used for the allowing the different frequency and power for getting the maximum receiving power. It is of two types upper band \& lower band, the upper band is having more TX frequency then the TX of lower and the RX of upper is the TX of lower and vice versa.

Antenna:-It act as transmitter which can transmit and receive the microwave signals.

\section{A. Working Principle:}

\section{System Modeling}

The Transmitter converts the source message into an electrical signal. The Transmitter is basically responsible for encoding the message and then this encoded message is multiplied by carrier frequency i.e. modulate the signal and then over the channel. At the receive end, the receiver demodulate the received signal and decode it and generate the original message. Minimal distortion at the receiver end is referred as a good communication property [3].

Communication System

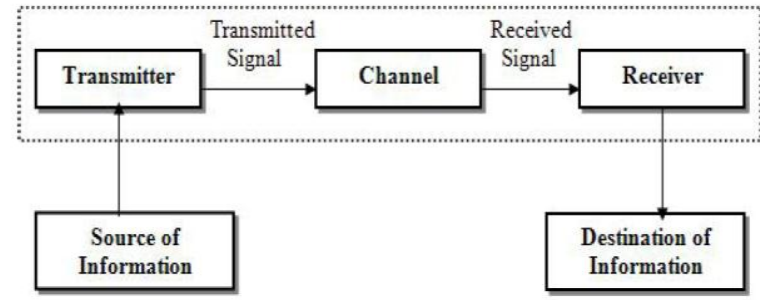

Figure 2.1 working of NEC microwave

\section{B. Microwave radio Link Design}

Following parameters should be considered for planning microwave radio link[11].

\section{Free Space Loss}

As signals spread out from a radiating source, the energy is spread out over a larger surface area. As this occurs, the strength of that signal gets weaker. Free space loss (FSL), measured in dB, and specifies how much the signal has weakened over a given distance. Figure 2.2 shows the formula to calculate FSL and what the theoretical loss would be at sample distances. The type of antenna used has no effect on FSL, since at any appreciable distance all antennas look like a point source radiator. Note that the difference in FSL between a $15 \mathrm{GHz}$ link and a $18 \mathrm{GHz}$ link is always about $1 \mathrm{~dB}$, regardless of the distance. 


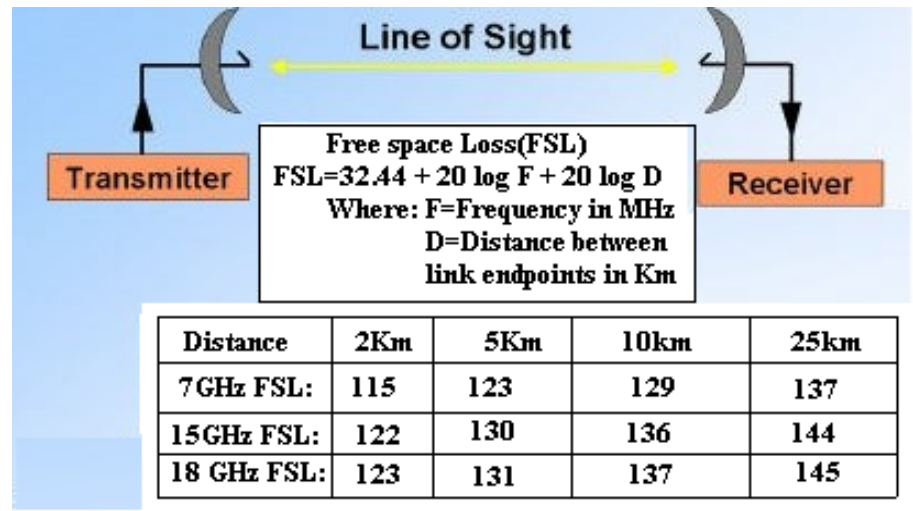

Figure 2.2 - Free Space Loss (FSL)

\section{Fresnel Zone}

Radio waves travel in a straight line, unless something refracts or reflects them. But the energy of radio waves is not "pencil thin." They spread out the farther they get from the radiating source - like ripples from a rock thrown into a pond. The area that the signal spreads out into is called the Fresnel zone (pronounced fra-nell). If there is an obstacle in the Fresnel zone, part of the radio signal will be diffracted or bent away from the straight-line path. The practical effect is that on a point-to-point radio link, this refraction will reduce the amount of RF energy reaching the receive antenna. The thickness or radius of the Fresnel zone depends on the frequency of the signal - the higher the frequency, the smaller the Fresnel zone. Figure 2.3 illustrates how the Fresnel zone is fattest in the middle. As with FSL, the antennas used have no effect on the Fresnel zone.

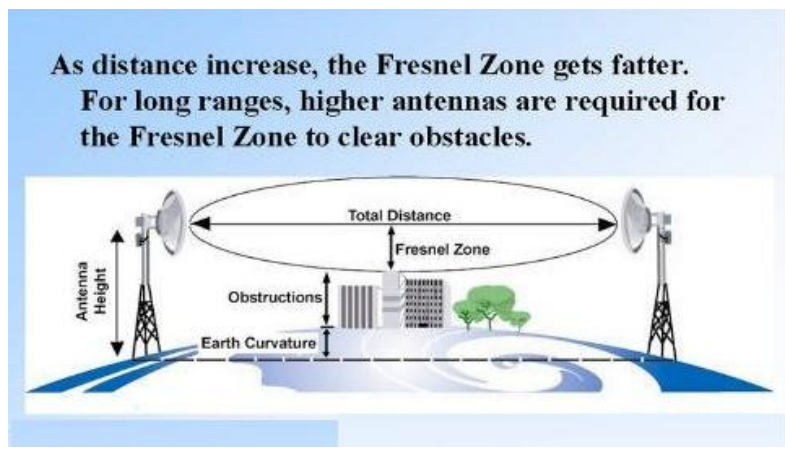

Figure 2.3 - Fresnel Zone

\section{Receive Signal Level}

Receive signal level is the actual received signal level (usually measured in negative $\mathrm{dBm}$ ) presented to the antenna port of a radio receiver from a remote transmitter.

\section{Receiver Sensitivity}

Receiver sensitivity is the weakest RF signal level (usually measured in negative $\mathrm{dBm}$ ) that a radio needs receive in order to demodulate and decode a packet of data without errors.

\section{Antenna Gain}

Antenna gain is the ratio of how much an antenna boosts the RF signal over a specified low-gain radiator. Antennas achieve gain simply by focusing RF energy. If this gain is compared with an isotropic (no gain) radiator, it is measured in $\mathrm{dBi}$. If the gain is measured against a standard dipole antenna, it is measured in $\mathrm{dBd}$. Note that gain applies to both transmit and receive signals.[6]

\section{Transmit Power}

The transmit power is the RF power coming out of the antenna port of a transmitter. It is measured in $\mathrm{dBm}$, Watts or milliWatts and does not include the signal loss of the coax cable or the gain of the antenna.

Effective Isotropic Radiated Power Effective isotropic radiated power (EIRP) is the actual RF power as measured in the main lobe (or focal point) of an antenna. It is equal to the sum of the transmit power into the antenna (in $\mathrm{dBm}$ ) added to the $\mathrm{dBi}$ gain of the antenna. Since it is a power level, the result is measured in 
$\mathrm{dBm}$. Figure 2.4 shows how $+24 \mathrm{dBm}$ of power $(250 \mathrm{~mW})$ can be "boosted" to $+48 \mathrm{dBm}$ or 64 Watts of radiated power.

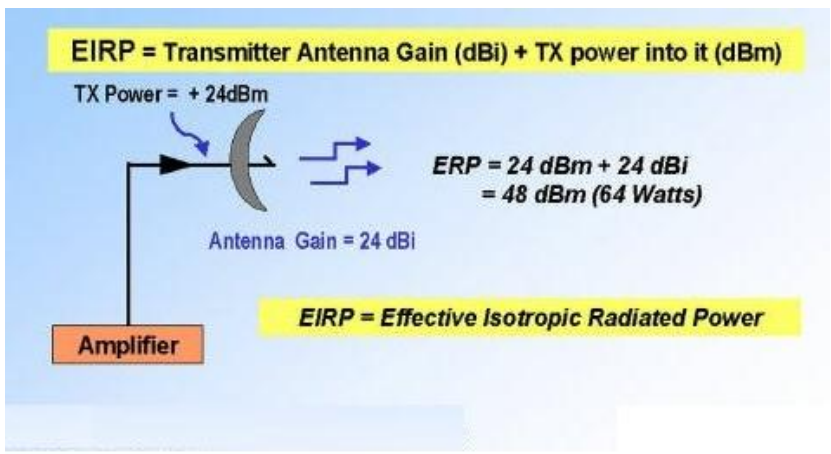

Figure 2.4 - Effective Isotropic Radiated Power (EIRP)

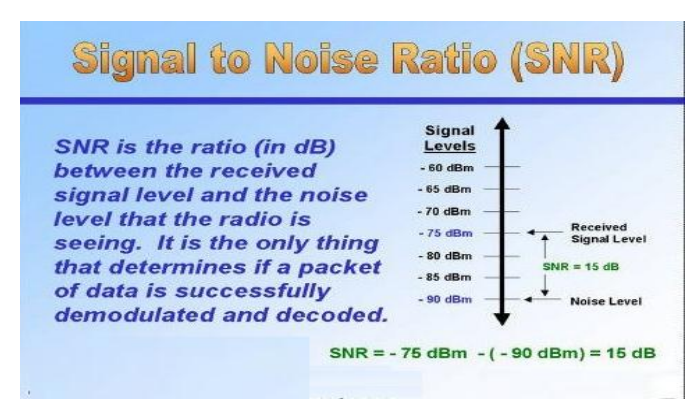

Figure 2.6 - Signal-to-Noise Ratio (SNR)

\section{System Operating Margin}

System operating margin ( $\mathrm{SOM}$ ) is the difference (measured in $\mathrm{dB}$ ) between the nominal signal level received at one end of a radio link and the signal level required by that radio to assure that a packet of data is decoded without error (see Figure 2.5). In other words, SOM is the difference between the signal received and the radio's specified receiver's sensitivity. SOM is also referred to as link margin or fade margin. [9]

\section{First Step in Planning a Link}

Whether your link is point-to-point or point-to-multipoint, the first thing to do is to verify that it will have not only clear line of sight, but at least 60 percent of the first Fresnel zone clear of obstructions as well. The longer the distance, the more important this is. If the Fresnel zone is blocked, then you will get a lower signal level on the distant end than expected - even if you can literally "see" the other antenna in the distance. But even if your Fresnel zone is partially blocked, it is still possible to get a link, provided that your system was designed to have a strong signal at the other end of the link. In planning long-range microwave links where you are not sure that you have unobstructed line-of-site and clear Fresnel zone, an RF path analysis should be done. There are many software packages available that have terrain data and can create a path profile from a set of latitude/longitude coordinates. But these programs can only indicate for certain if a link will not work due to terrain obstruction. A clear path on paper is not a guarantee that your link will work, since it does not show trees or buildings. So even a "clear" link might have 80-foot trees in the way that could block the signal. You could be wasting your time and money if you ignore Fresnel zone issues — or worse yet, no line-of-site — and attempt to set up a link anyway. You will likely not have a reliable link, if one at all. But assuming that you do have clear line-of-site and 60 percent of the first Fresnel zone clear (or nearly clear), how can you know if you will have a good link or not? How much gain do your antennas need to have?

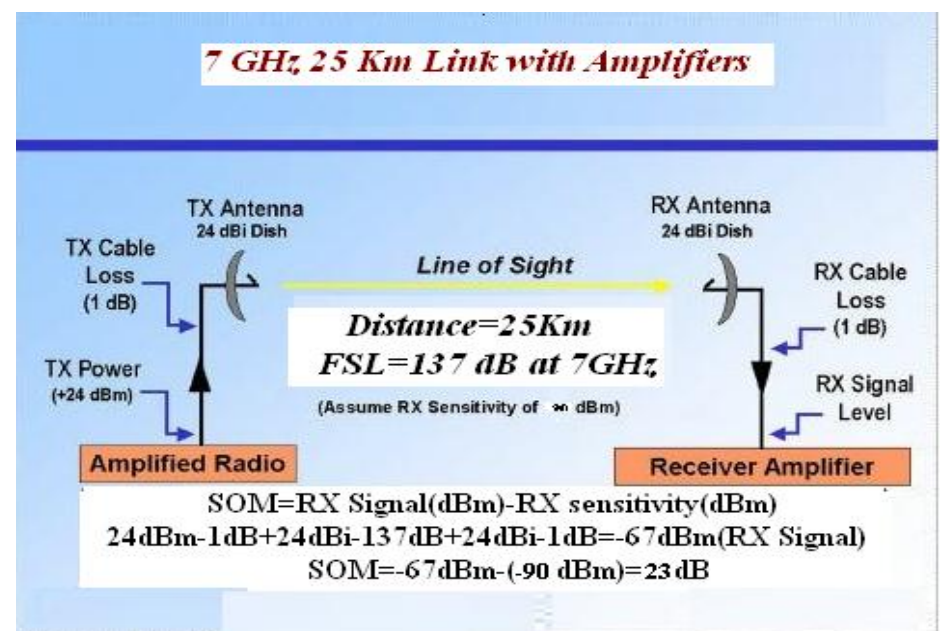

Figure 2.5 - System Operating Margin (SOM)

\section{Multipath Interference}

When signals arrive at a remote antenna after being reflected off the ground or refracted back to earth 
from the sky (sometimes called ducting), they will subtract (or add) to the main signal and cause the received signal to be weaker (or stronger) throughout the day.

\section{Signal-to-Noise Ratio}

Signal-to-noise ratio (SNR) is the ratio (usually measured in $\mathrm{dB}$ ) between the signal level received and the noise floor level for that particular signal. The SNR is really the only thing receiver demodulators really care about. Unless the noise floor is extremely high, the absolute level of the signal or noise is not critical. Figure 2.6 illustrates that weaker signals are larger negative numbers. It also graphically shows how the SNR is computed.

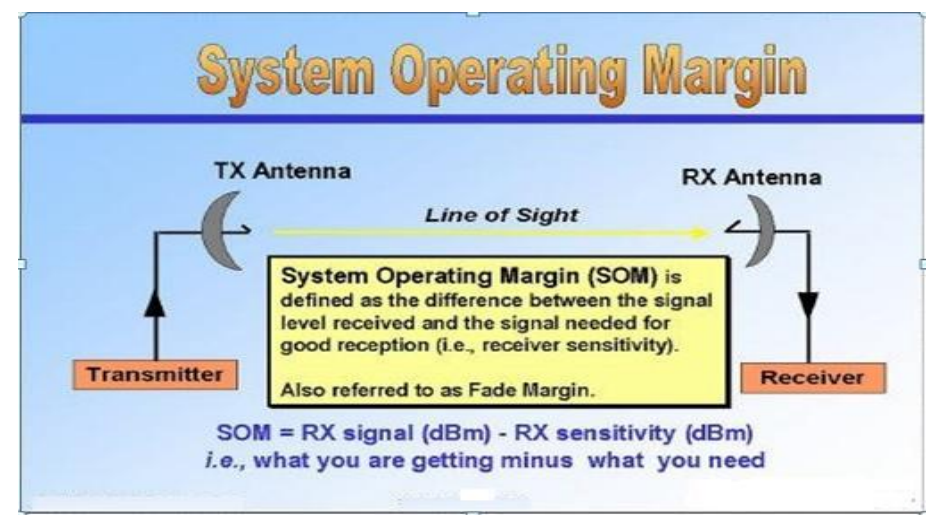

Figure 2.7 Sample System Operating Margin (SOM) Calculation

much coax cable loss is too much? If your link is at $2.4 \mathrm{GHz}$, should external amplifiers be used? Or given your fixed base station antenna with a pre-set gain, how far can you reach with the different types of client antennas? And which clients will need amplification?

\section{Why Perform an SOM Calculation?}

By doing an SOM calculation, we can test various system designs and scenarios to see how much fade margin (or "safety cushion") our link will theoretically have. Figure $\mathbf{2 . 7}$ illustrates a sample SOM calculation on a point-to-point link. It presumes that the antennas are aimed at each other properly (i.e., they are in each others' main lobe). To calculate SOM in the example, start with the transmit power $(+24 \mathrm{dBm})$, subtract the coax cable loss $(1 \mathrm{~dB})$, and add the transmit antenna gain $(24 \mathrm{dBi})$. This gives you the effective isotropic radiated power[5,11]:

EIRP $=$ TX Power - Coax Cable Loss + TX Antenna Gain.

Then subtract the FSL (137 dB), add the receiver antenna gain ( $24 \mathrm{dBi})$, subtract the coax cable loss $(1 \mathrm{~dB})$ and you get the signal reaching the receiver:

RX Signal $=$ EIRP - FSL + RX Antenna Gain - Coax Cable Loss

Compute the difference between the received signal and the radio's receiver sensitivity to determine the SOM. In this example, the received signal is $-67 \mathrm{dBm}$ and the receiver's sensitivity is $-90 \mathrm{dBm}$ giving a theoretical SOM of $23 \mathrm{~dB}$

\section{Proposed system}

1) Controlling power of IDU by sensing water drop

2) Control of Diesel Generator(DG) by using auxiliary port of IDU

3) Adding cooling fan

4) Status of alarms on Mobile by sending SMS

1) Block Diagram of controlling power of IDU 


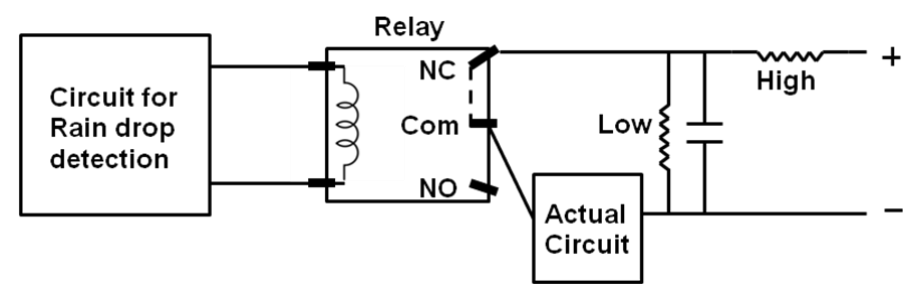

Figure 2.8 Power controlling unit

1) First sensing water drop on the surface of the IDU, the output of the water drop sensor is connected to the relay as a input, and power of IDU is connected to IDU through relay (NC terminal) and RC circuit, thus when water falls on the surface of the sensor it gives input to the relay hence coil of relay becomes active and it power off the IDU, when water drop sensor sense water is not falling it does not gives any output and hence relays NC terminal becomes active and hence

2) Control of Diesel Generator by using auxiliary port of IDU, connecting the alarm cable to the auxiliary port of the IDU, when AC power cutoff alarm cable send the signal to the auxiliary port of the IDU and the it will power ON the DG.

3) Adding two cooling fan for avoiding the problem of high temperature.

4) Which alarms are generate are send on mobile to take the design of which troubleshooting methods to be used for it to take action fast against it to reduced the troubleshooting time, To perform all this operation I am using 16 bit AVR \& GSM modem.

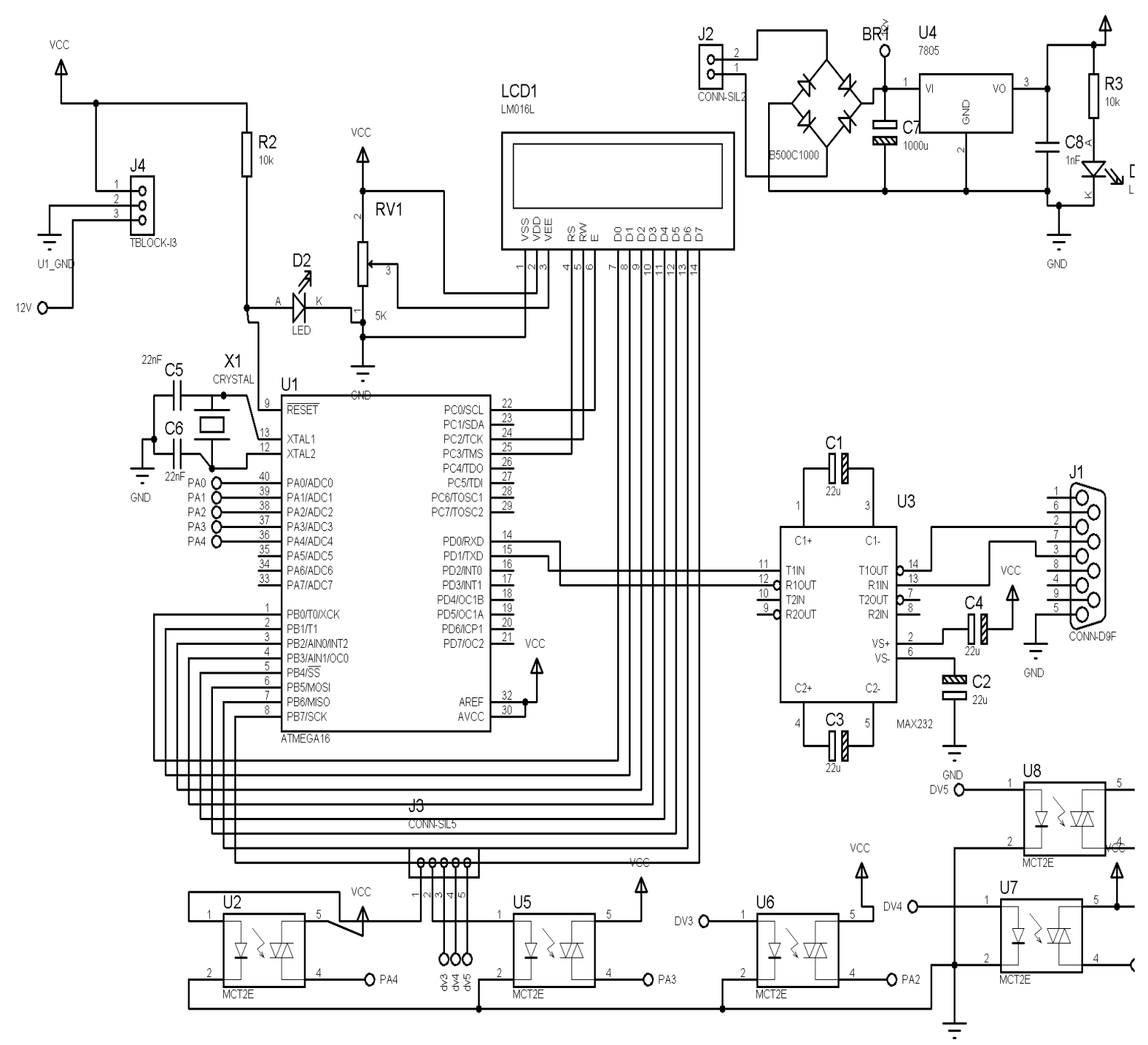

Figure 2.9 Circuit Diagram of proposed system 


\section{Performance Analysis}

A. Flowchart for Reducing time of troubleshooting
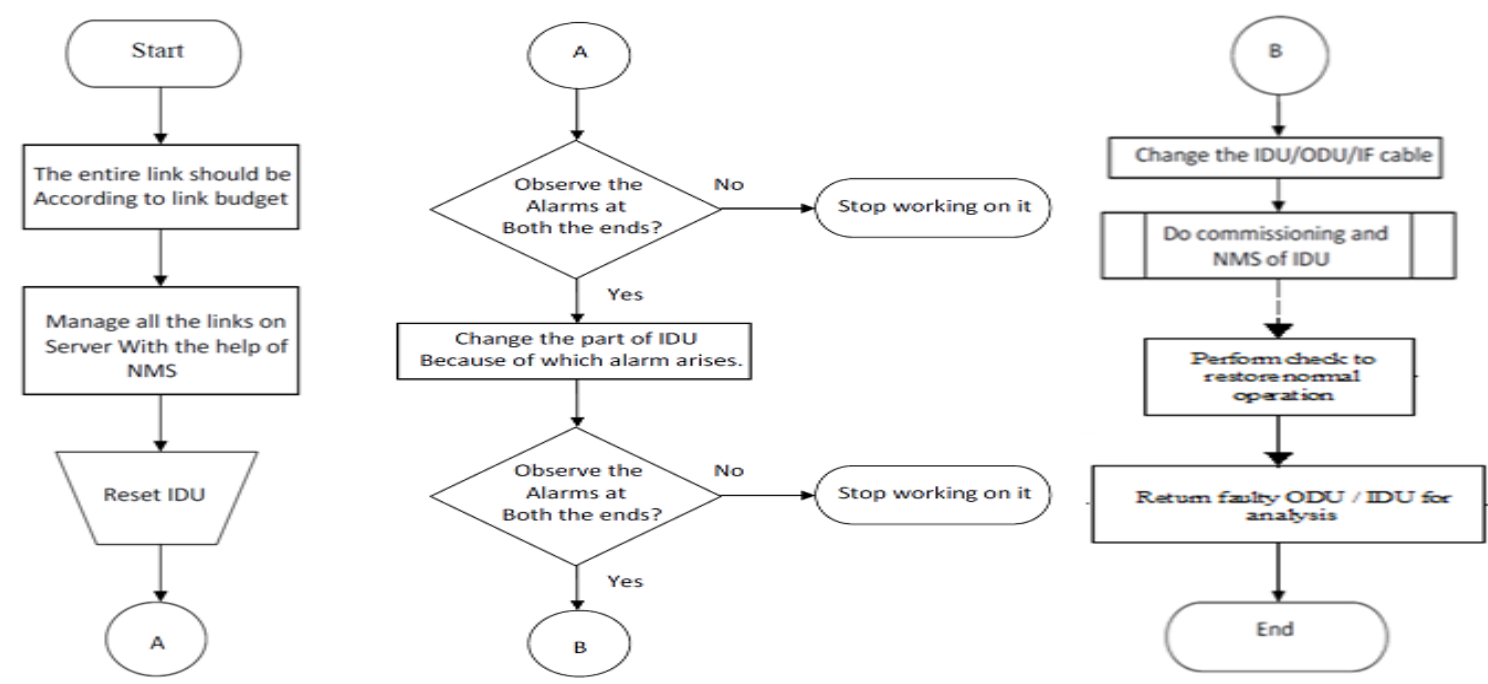

Figure:-3.1 Flow chart of trouble shooting Method.

Figure 3.1 shows the general flow chart, which can be used for any equipment(Other then NEC) \& for any operator(Other then TATA), the main aim of this flow chart is to reduce the call drops of any operators, and if drop occurs how to overcome the drop within very small time spectrum.

First stage (Start-A) are written to avoid the call drops, from second stage (A-End) if call drop occurs how to overcome that drop is mention, because many operators are using NEC equipment In microwave that's why it will be helpful to all the operators, near about four operators are dependent on TATA that's why it is more useful for TATA.

The following no of operators are using NEC

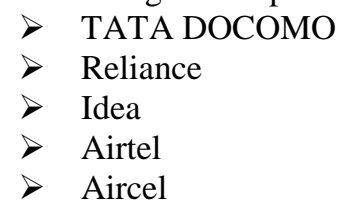

Only Vodafone and BSNL is not using this equipment they are using NOKIA, but it will be helpful for nokia employee also for their troubleshooting method [4].

\section{B. Description of Acknowledgment, Acknowledgment And their troubleshooting Methods}

1) Description(Figure 3.2)

In this four alarms is generated, details are as follows
a) EARLY WARNING
b) FRAME ID
c) $\mathrm{LOF}$
d) HIGH BER

Bit Error Rate (BER) is the percentage of bits that have errors relative to the total number of bits received in a transmission, usually expressed as ten to a negative power. For example, a transmission might have a BER of 10 to the minus 6 , meaning that, out of 1,000,000 bits transmitted, one bit was in error. The definition of bit error rate can be translated into a simple formula:

\section{BER = number of errors / total number of bits sent}

If the medium between the transmitter and receiver is good and the signal to noise ratio is high, then the bit error rate will be very small - possibly insignificant and having no noticeable effect on the overall system However if noise can be detected, then there is

Chance that the bit error rate will need to be considered.[5,6] 
Early Warning:- This alarm is generated due to the increase in Bit Error Rate(BER), it is notified that the continuous increment in BER creates complications in link.

At the starting stage of BER, it generates early warning alarm and a increment of BER results in following alarms

- Low BER

- High BER

- DEM Alarm

Frame ID:- (It is the application which is inserted by NEC to avoid the interference in between the two link) This alarm is generated when there is mismatch of frame ID between the two sites of single link.

LOF(Loss Of frame):-when any tree, building, any other radio, etc comes in between the two microwave of single link then this alarm will appear. in LOF when we transmit signal does not reach at the receiver end and return to the transmitter.

High BER:-when signal to noise ratio decreases it tends to increase in BER and due to increase in bit error rate high $\mathrm{BER}$ is generated.

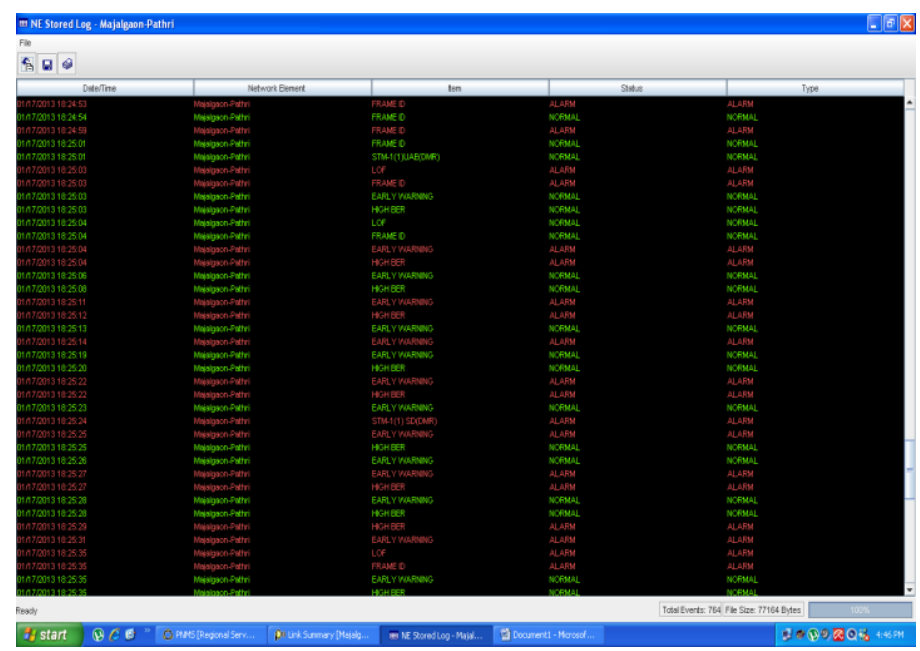

Figure 3.2 Acknowledgment 1

\section{Troubleshooting}

When all this alarm comes together then it means there is a major chance of interference or misalignment (To check whether interference exist or not, disconnect power supply at one end and check the RX level at other end and vice versa, if RX level is coming in the range of -99 to $-80 \mathrm{dbm}$ at both the end then there is no interference)

If Interference:-we need to change the frequency $\&$ frame ID at both the end

If misalignment:-We need to make the proper alignment at both the ends.

\section{2) Description(Figure 3.3)}

In this following alarm are observed

a) RX Level

b) $\mathrm{LOF}$

c) STM-1(1)(DMR)

d) TCN-RX LEV-15min

RX Level:-This alarm is generated when receiving level decreases from its limit.

LOF:-when there is any frame loss takes place then this alarm will appear.

STM-1(1)(DMR):-when there is no transmission through optical port then this alarm will appear.

TCN-RX LEV-15min:-when the RX level reduces from their limit after 15 mints this alarm will appear.

\section{Troubleshooting}

When all this alarm comes together then it means there is a major chance of power failure at far end.

The power failure may occurs due to following reasons

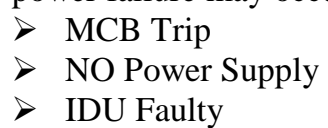

If MCB is Trip:-Then either change the MCB or repair the MCB

If No power Supply:-Make the arrangement of power supply 
If IDU faulty:-please check the power card if not working change the power card and if it may creating problem the changed the complete IDU.

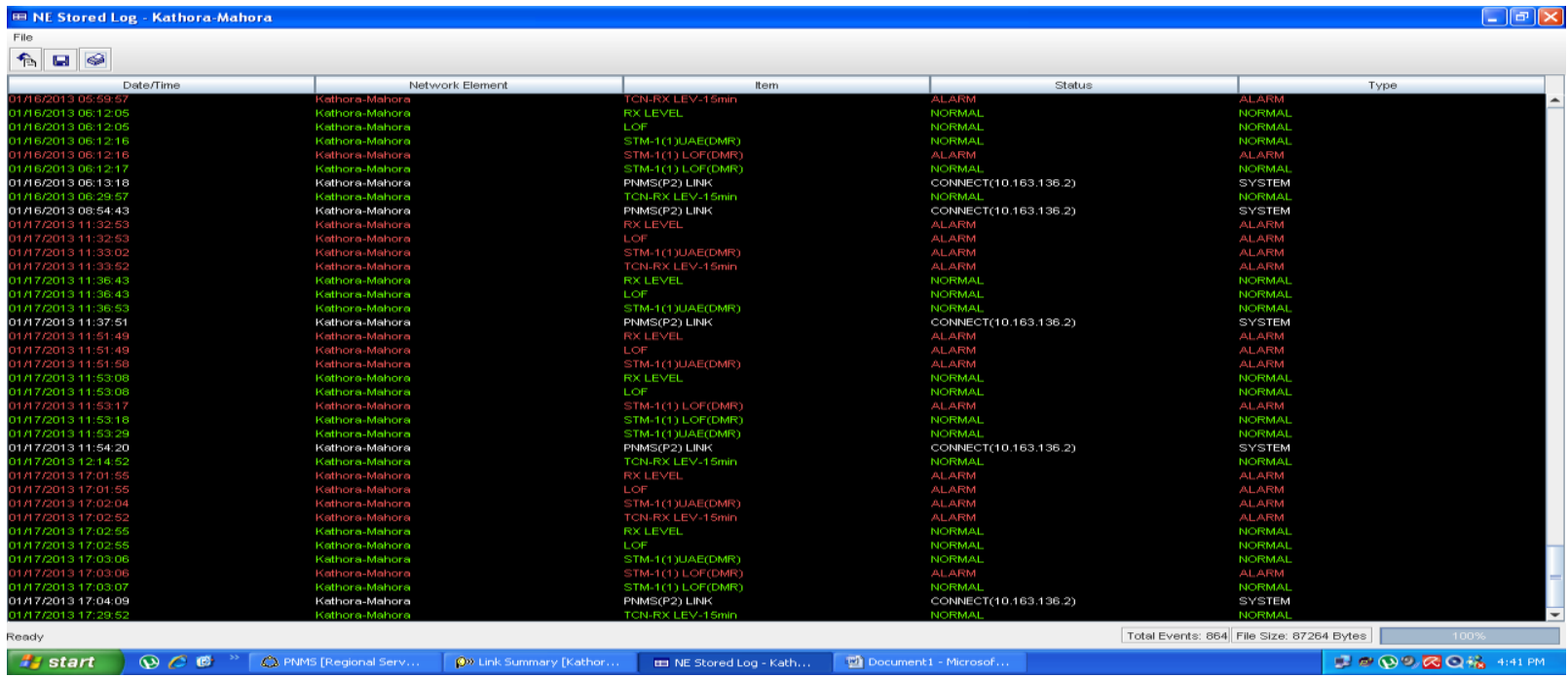

Figure 3.3 Acknowledgment

\section{Report of Breakdown Maintenance Activity carried out}

\section{Table 4.1 Breakdown Report}

\begin{tabular}{|c|c|c|c|c|c|c|c|c|c|c|c|c|c|}
\hline \multirow[t]{2}{*}{ No. } & \multirow[t]{2}{*}{ Location } & \multirow[t]{2}{*}{ Hop Name } & \multicolumn{2}{|c|}{$\begin{array}{l}\text { Time of Link } \\
\text { failure }\end{array}$} & \multicolumn{2}{|c|}{$\begin{array}{l}\text { Time of being } \\
\text { informed of link } \\
\text { failure by TTML }\end{array}$} & \multicolumn{2}{|c|}{$\begin{array}{c}\text { Time of reaching the } \\
\text { site }\end{array}$} & \multirow{2}{*}{\begin{tabular}{|c|} 
Time \\
spent in \\
travel to \\
Site
\end{tabular}} & \multirow{2}{*}{$\begin{array}{c}\text { Time for } \\
\text { arranging } \\
\text { spares (if } \\
\text { applicable) }\end{array}$} & \multirow{2}{*}{$\begin{array}{c}\text { Time for } \\
\text { site access } \\
\text { permission } \\
\text { (if } \\
\text { applicable) }\end{array}$} & \multicolumn{2}{|c|}{$\begin{array}{c}\text { Time when link was } \\
\text { restored }\end{array}$} \\
\hline & & & Date & Time & Date & Time & Date & Time & & & & Date & Time \\
\hline 1 & Gangapur & Mahalgaon - Gangapur & $14 / 10 / 2013$ & 7:55PM & $14 / 10 / 2013$ & 8:00PM & $14 / 10 / 2013$ & 9:30PM & 1.5 Hours & NA & NA & $14 / 10 / 2013$ & 09:40PM \\
\hline 2 & Aurangaba & Sarafa Bazar - VK Market & $15 / 10 / 2013$ & 6.55PM & $15 / 10 / 2013$ & 7:00PM & $15 / 10 / 2013$ & 7:20PM & 20 minute & NA & NA & $15 / 10 / 2013$ & $07: 35 \mathrm{PM}$ \\
\hline 3 & Aurangaba & N11 Ravi Nagar - N12 & $23 / 10 / 2013$ & 6.25PM & $23 / 10 / 2013$ & 6:30PM & $23 / 10 / 2013$ & 7:00PM & 30 Minute & NA & NA & $23 / 10 / 2013$ & 7:20PM \\
\hline 4 & Aurangaba & SRPF - Sataragaon & $29 / 10 / 2013$ & 10:35AM & $29 / 10 / 2013$ & 10:40AM & $29 / 10 / 2013$ & 11:20AM & 30 Minute & NA & NA & $29 / 10 / 2013$ & 11:30AM \\
\hline 5 & Jalna & Nutan Vasahat - Nariman Nagar & $2 / 11 / 2013$ & 5.55AM & $2 / 11 / 2013$ & 6:00AM & $2 / 11 / 2013$ & 7:00AM & 1 Hours & NA & NA & $2 / 11 / 2013$ & 7:20AM \\
\hline 6 & Jalna & jafrabad - Dudha & $7 / 11 / 2013$ & 4.55AM & $7 / 11 / 2013$ & 5:00AM & $7 / 11 / 2013$ & 7:00AM & 2 Hours & NA & NA & $7 / 11 / 2013$ & 7:30AM \\
\hline 7 & Bhokardan & Walsangwi Excel - Shivna & $7 / 11 / 2013$ & 7:50PM & $7 / 11 / 2013$ & 8:00PM & $7 / 11 / 2013$ & 9:30PM & 1.5 Hours & NA & NA & $7 / 11 / 2013$ & 10:00PM \\
\hline 8 & Beed & Talawada - Tirthapuri & $12 / 11 / 2013$ & 8:35PM & $12 / 11 / 2013$ & 8:40PM & $12 / 11 / 2013$ & 10:40PM & 2 Hours & NA & NA & $12 / 11 / 2013$ & 11:00PM \\
\hline 9 & Beed & Talawada - Tirthapuri & $13 / 11 / 2013$ & 5.50AM & $13 / 11 / 2013$ & 6:00AM & $13 / 11 / 2013$ & 8:00AM & 2 Hours & NA & NA & $13 / 11 / 2013$ & 08:10AM \\
\hline 10 & Beed & Shahagad - Talawada & $13 / 11 / 2013$ & 5:05PM & $13 / 11 / 2013$ & 5:10PM & $13 / 11 / 2013$ & 6:10PM & 1 Hours & NA & NA & $13 / 11 / 2013$ & $6: 15 \mathrm{PM}$ \\
\hline 11 & Beed & Shahagad - Talawada & $14 / 11 / 2013$ & 5:35AM & $14 / 11 / 2013$ & 5:40AM & $14 / 11 / 2013$ & 7:40AM & 2 Hours & NA & NA & $14 / 11 / 2013$ & 07:50AM \\
\hline 12 & Jintur & Shivdi Rptr - Jintur & $15 / 11 / 2013$ & 12:10AM & $15 / 11 / 2013$ & 12:10AM & $15 / 11 / 2013$ & 3:10AM & 3 Hours & NA & NA & 3:20AM & 3:20AM \\
\hline
\end{tabular}

Table 4.1 shows the breakdown report of maintenance carried out at tata docomo Aurangabad, the report shows the details of 12 link failures and the details of date and time of link failure, when we reached the site and when site was restored. In each sites we can see the troubleshooting is done within 30 minutes. And in SrNo:8,9 \& 10,11 the same link failures occurs two time to avoid this proposed system is developed and I am sure if we used the proposed system then the numbers of breakdown will be reduced.

\section{Comparison of existing system and proposed system}

\begin{tabular}{|l|l|l|l|}
\hline Sr No & Parameters & \multicolumn{1}{|c|}{ Existing system } & Proposed system \\
\hline 1 & Cost of single IDU & 25000 & 30000 \\
\hline 2 & L*H*B & $48.9 \mathrm{~cm}^{*} 18.7 \mathrm{~cm}^{*} 3.7 \mathrm{~cm}$ & $48.9 \mathrm{~cm}^{*} 18.7 \mathrm{~cm}^{*} 6.4 \mathrm{~cm}$ \\
\hline 3 & Total cost of link & 2.4 lacks & 2.5 lacks \\
\hline 4 & Troubleshooting time & Larger then proposed system & Less then the existing system \\
\hline 5 & Availability & Easily available in market & Need to manufacture \\
\hline 6 & Advantages & Low price, easily available, small size & $\begin{array}{l}\text { No power fluctuations, no problem of high } \\
\text { temperature, DG can be control with IDU and } \\
\text { troubleshooting time is reduced because } \\
\text { system is work on SMS. }\end{array}$ \\
\hline 7 & Disadvantages & $\begin{array}{l}\text { No cooling fans hence Temperature } \\
\text { problem, power card faulty because of } \\
\text { heavy rain, no control of DG, no sms } \\
\text { indicator to troubleshoot online }\end{array}$ & $\begin{array}{l}\text { The size is big, cost in increased, if equipment } \\
\text { become faulty then we need to transfer all the } \\
\text { circuits into the new system. }\end{array}$ \\
\hline
\end{tabular}




\section{Conclusion}

By the sanctity of caller and callee voice information, we have reduced the microwave acknowledgement with the particular alarm at particular frame, by providing the proper troubleshooting command action with respective to the feedback error signal. Due to minimization of microwave error signal we can improve the quality of microwave signal and reducing the call dropping between caller and callee.

\section{References}

[1]. Md. Rakib Al Mahmud, “Analysis And Planning Microwave Link To Established Efficient Wireless Communications”, September 2009.

[2]. J. Frank Jimene, 1999, "Fundamentals of Radio Link Engineering".

[3]. H. Abdullah Jr, Paulo Carvalho, Luis. F. Molinaro, Carlos Evangelista, L Bermudez, "Tools For Microwave Radio Communications System Design", April 2003.

[4]. "Basic Path Considerations For A Microwave Link", May 2010, EM Clarity White Paper.

[5]. Website of NEC Corporation, Japan.

[6]. Sanjeeva Gupta, 1957, Microwave Engineering. McGraw-Hill

[7]. William Stalling, “Wireless Communications \& Networks”, 2nd ed, Prentice,Hall,2005

[8]. Telecom Regulatory Authority of India.

[9]. Practical telecommunications and wireless communications for business AvEdwin Wright, Deon Reynders [Online Book].

[10]. Website of ceragaon

[11]. www.ydi.com 